HKU CSIS Tech Report TR-2002-12

\title{
Computing Real Inflection Points of Cubic Algebraic Curves
}

\author{
Falai Chen \\ Department of Mathematics \\ University of Science and Technology of China \\ Hefei, Anhui 230026, P. R. China \\ Email: chenfl@ustc.edu.cn \\ Wenping Wang * \\ Department of Computer Science and Information System \\ University of HongKong \\ HongKong, P. R. China \\ Email: wenping@csis.hku.hk
}

\begin{abstract}
Shape modeling using planar cubic algebraic curves calls for computing the real inflection points of these curves since an inflection point represents important shape feature. A real inflection point is also required for transforming projectively a planar cubic algebraic curve to the normal form, in order to facilitate further analysis of the curve. However, the naive method for computing the inflection points of a planar cubic algebraic curve $f=0$ by directly intersecting $f=0$ and its Hessian curve $H(f)=0$ requires solving a degree nine univariate polynomial equation, and thus is not efficient. In this paper we present an algorithm for computing the real inflection points of a planar cubic algebraic curve. The algorithm follows Hilbert's solution for computing the inflection points of a cubic algebraic curve in the complex projective plane. Hilbert's solution is based on invariant theory and requires solving only a quartic polynomial equation. Through a detailed study with emphasis on the distinction between real and imaginary inflection points, we adapt Hilbert's solution to efficiently compute only the real inflection points of a cubic algebraic curve $f=0$, without exhaustive but unnecessary search and root testing. To compute the real inflection points of $f=0$, only two cubic polynomial equations need to be solved in our algorithm and it is unnecessary to solve numerically the quartic equation prescribed in Hilbert's solution. In addition, the invariants of $f=0$ are used to analyze the singularity of a singular curve, since the number of the real inflection points of $f=0$ depends on its singularity type.
\end{abstract}

Key words: algebraic curve, cubic curve, Hessian curve, singular point, inflection point, invariant

${ }^{*}$ Corresponding author. 


\section{Introduction}

An inflection point, or flex, of a planar algebraic curve $f(x, y, w)=0$ is a simple point of $f=0$ at which the tangent of $f=0$ has at least an order three contact with $f=0$. Algebraically, the inflection points of an algebraic curve $f=0$ are at the intersection of $f=0$ with the Hessian curve $H(f)=0$ of $f=0$. This article is about computing the real inflection points of an irreducible planar cubic algebraic curve.

Planar cubic algebraic curves are the simplest planar curves containing inflection points in [Walker, 1950; Patterson, 1988b]. Shape modeling with planar cubic algebraic curves have been studied in [Paluszny and Patterson, 1998]. Inflection points are of interest in this setting since it is often necessary to know if a given cubic curve segment contains an inflection point.

A real inflection point is also useful when one needs to map projectively a planar cubic algebraic curve to a the normal form $y^{2}=g(x)$ (i.e. the Weierstrass form), where $g(x)$ is a cubic polynomial in $x$, since the normal form can be obtained by placing a real inflection point of $f=0$ at the point $(0,1,0)$ at infinity. The normal form of a planar cubic facilitates the study of the projectively invariant properties and the parameterization of the curve. Such an application of planar cubic curves is considered in [Patterson, 1988a] for parameterizing a planar cubic and in [Wang et al, 2002] for analyzing the intersection curve between two quadric surfaces in 3D space, which is shown to be related birationally to a planar cubic curve. Without using an inflection point, one normally would have to use a more involved birational quadratic transformation to reduce a planar cubic to the normal form [Abhyankar and Bajaj, 1987; Patterson, 1988a].

Most of existing research in CAGD on computing inflection points consider rational parametric curves. The detection of inflection points on a rational planar parametric cubic curve, which is planar singular cubic algebraic curve, is studied in [Wang 1981; Stone and DeRose, 1989]. The detection and computation of inflection points of general rational parametric curves are studied in [Manocha and Canny, 1992; Li and Cripps, 1997].

Given a planar algebraic cubic $f=0$, its Hessian curve $H(f)=0$ is also a cubic curve. Hence, in general, a planar cubic curve has nine inflection points, including real and complex conjugate ones. For a real nonsingular cubic curve, these nine inflection points comprise three real inflection points and six imaginary ones [Walker, 1950]. These inflection points can be computed by directly intersecting $f=0$ with $H(f)=0$, which requires solving a univariate equation of degree nine. Probably due to the lack of emphasis of classical algebraic geometry on the computation aspect of algebraic curves, it has been unclear to the CAGD community whether the inflection points of a planar cubic can be found more easily than solving a degree nine equation [Patterson, 1988a]. However, the existence of a simpler solution seems to be possible,

since it is well known that the nine inflection points of a planar cubic form a so-called nine-point configuration, i.e. every line passing through two of the nine points also passes through a third 
[Walker, 1950].

Indeed, such a solution was given by Hilbert more than a hundred years ago [Hilbert, 1993]. As an application of his work on invariant theory, Hilbert presented a method for computing the inflection points of a planar cubic in the complex plane through solving a quartic equation, called the characteristic equation. In this paper we adapt Hilbert's solution to devise an efficient algorithm for computing only the real inflections of a real cubic algebraic curve.

Since often only real inflection points are of concern in CAGD, we study the ramifications of distinguishing real and imaginary inflection points. If only real inflections are sought, a direct implementation of Hilbert's solution would be inefficient since it would require exhaustive search and computation with a number of imaginary roots of a quartic equation as well as the imaginary linear components of a reducible cubic curve. Our goal in this paper is to adapt Hilbert's solution to efficiently compute the real inflection points of a cubic algebraic curve.

Specifically, our contributions are: (1) We show how to use invariants to detect singular cubics in order to compute real inflection points for different types of cubics, since the number of the real inflection points depends on the singularity type of a cubic curve; (2) We show that all the real inflection points of a cubic algebraic curve lie on a real line given by a particular real root of the quartic characteristic equation of Hilbert; (3) We give a simple closed-form formula for this particular real root; hence, the real inflection points of a cubic algebraic curve can be computed without having to solve numerically Hilbert characteristic equation of degree four. Only two

cubic polynomial equations need to be solved in a step for extracting a real linear component of a reducible cubic algebraic curve in our algorithm.

The remainder of this paper is organized as follows. In Section 2 preliminaries about planar cubic curves and invariants of a ternary form are introduced. In Section 3 we present the algorithm for computing singular points and real inflection points of a planar real cubic algebraic curve.

\section{Preliminaries}

A planar algebraic curve $f(x, y, w)=0$ of degree $n$ is given by the ternary form

$$
f(x, y, w):=\sum_{i, j, k \geq 0, i+j+k=n} f_{i j k} x^{i} y^{j} w^{k}
$$

For a cubic curve $f=0$ to be considered in this paper, we assume throughout that the coefficients $f_{i j k}$ are real, and we call $f=0$ a planar real cubic algebraic curve, or a cubic curve for short when there is no danger of confusion.

An algebraic curve $f=0$ is reducible if $f$ can be factored into two lower degree polynomials, that is, there exist polynomials $f_{1}(x, y, w)$ and $f_{2}(x, y, w)$ such that $f=f_{1} f_{2}$. Otherwise, $f=0$ 
is irreducible. Geometrically, a reducible curve comprises some components of lower degree algebraic curves.

A point $\left(x_{0}, y_{0}, z_{0}\right)$ is a singular point of a planar algebraic curve $f(x, y, z)=0$ if

$$
f_{x}\left(x_{0}, y_{0}, w_{0}\right)=f_{y}\left(x_{0}, y_{0}, w_{0}\right)=f_{w}\left(x_{0}, y_{0}, w_{0}\right)=0 .
$$

By Euler's identity $n f=x f_{x}+y f_{y}+z f_{z}$, these conditions imply that $\left(x_{0}, y_{0}, w_{0}\right)$ is a point on $f=0$. The curve $f=0$ is called singular if it has a singular point; otherwise, it is called nonsingular. A singular point $p$ is characterized geometrically by that the tangent to the curve $f=0$ at $p$ is not uniquely defined.

An inflection point of the curve $f=0$ is a nonsingular point $p$ on $f=0$ at which the tangent to $f=0$ has at least an order three contact with $f=0$ [Walker, 1950]. The inflection points of the curve $f=0$ can be determined by the intersection of $f=0$ and its Hessian curve

$$
H(f):=\left|\begin{array}{ccc}
\frac{\partial^{2} f}{\partial x^{2}} & \frac{\partial^{2} f}{\partial x \partial y} & \frac{\partial^{2} f}{\partial x \partial w} \\
\frac{\partial^{2} f}{\partial y \partial x} & \frac{\partial^{2} f}{\partial y^{2}} & \frac{\partial^{2} f}{\partial y \partial w} \\
\frac{\partial^{2} f}{\partial w \partial x} & \frac{\partial^{2} f}{\partial w \partial y} & \frac{\partial^{2} f}{\partial w^{2}}
\end{array}\right|=0 .
$$

For a cubic curve $f=0, H(f)=0$ is also a cubic curve. Thus, in general, a cubic curve $f=0$ has nine infection points (real or imaginary), since, by Bézout's theorem, two cubic curves have nine intersections, unless they have a common component. However, $f=0$ has fewer inflection points when it is reducible or singular,

The Hessian curve $H(f)=0$ can be used to detect whether a cubic curve is reducible or not.

Proposition 1 (1) A cubic curve $f=0$ consists of three lines if and only if its Hessian curve $H(f)=0$ and $f=0$ are the same curve. (2) A cubic curve $f=0$ consists of a line and a proper conic if and only if $f=0$ and $H(f)=0$ have a common line.

Proof: Assume that the curve $f=0$ consists of three lines. Then $f=l_{1} l_{2} l_{3}$ for three homogenous linear factor $l_{i}(x, y, w), i=1,2,3$. Since every point of $f=0$ is either a singular point or a point on a line, $H(f)$ vanishes at every point of $f=0$. Thus $H(f)=c l_{1} l_{2} l_{3}$ for some constant $c$. Conversely, if there exists some constant $c$ such that $H(f)=c f$, then every point on the curve $f=0$ is an inflection point. But this occurs only when $f=0$ degenerates to three lines. The first part of the proposition is proved.

For the second part of the proposition, assume $f=l C$, where $l$ is a linear factor and $C$ is a quadratic factor. Direct calculation shows $H(f)=l C^{\prime}$, where $C^{\prime}$ is a quadratic function. Hence $f=0$ and $H(f)=0$ share a common line. Conversely, if $H(f)=0$ and $f=0$ have a common line $l=0$, then $l$ is a factor of $f$. But since $f$ cannot be factored into three linear factors, $f=0$ degenerates into a line and a conic curve. 
The above proposition gives an algorithm for detecting whether a cubic curve is reducible or not. First compute the Hessian curve $H(f)=0$ and check if there exists a constant $c$ such that $H(f)=c f$. If yes, $f=0$ consists of three lines. Otherwise, compute $h=G C D(f, H(f))$. If $h$ contains a linear factor, then $f=0$ reduces to a line and a proper conic. Otherwise, $f=0$ is irreducible. Hence, we assume hereafter that the cubic algebraic curve we will consider is irreducible.

An irreducible cubic curve can be singular or nonsingular. A singular cubic curve has one singular point, which is a double point of one of the following three types: crunode, cusp, and acnode [Walker, 1950]. As we will see later in the next section, a singular point of $f=0$ is also a singular point of its Hessian curve $H(f)=0$. Thus the number of inflection points of a singular cubic curve is less than nine.

A degree nine equation needs to be solved if one attempts to compute the inflection points of a cubic $f=0$ by directly intersecting $f=0$ with its Hessian curve $H(f)=0$. In contrast, Hilbert gives a solution based on invariant theory to the computation of the inflection points of a cubic that needs to solve some univariate quartic equations of degree four or less. To introduce Hilbert's solution, we first review some basic concepts from invariant theory. Two references on invariant theory are [Hilbert, 1993; Olver, 1999].

Let

$$
F\left(x_{1}, \ldots, x_{m}\right)=\sum_{i_{1}+\ldots+i_{m}=n} a_{i_{1} \ldots i_{m}} x_{1}^{i_{1}} \ldots x_{m}^{i_{m}}
$$

be a homogenous polynomial in $m$ variables. $F$ is also called a form. Consider an arbitrary linear transformation

$$
\left\{\begin{array}{cc}
x_{1}= & \alpha_{11} x_{1}^{\prime}+\ldots+\alpha_{1 m} x_{m}^{\prime} \\
\ldots & \ldots \\
x_{m}= & \alpha_{m 1} x_{1}^{\prime}+\ldots+\alpha_{m m} x_{m}^{\prime}
\end{array}\right.
$$

Assume the transformation is invertible, that is, the determinant

$$
\delta=\left|\begin{array}{ccc}
\alpha_{11} & \ldots & \alpha_{1 m} \\
\vdots & \vdots & \vdots \\
\alpha_{m 1} & \ldots & \alpha_{m m}
\end{array}\right| \neq 0
$$

After the above transformation, the form $F$ changes into another form of the same degree

$$
F^{\prime}\left(x_{1}^{\prime}, \ldots, x_{m}^{\prime}\right)=\sum_{i_{1}+\ldots+i_{m}=n} a_{i_{1} \ldots i_{m}}^{\prime}\left(x_{1}^{\prime}\right)^{i_{1}} \ldots\left(x_{m}^{\prime}\right)^{i_{m}}
$$

An invariant of form $F$ is a polynomial $I$ of the coefficients $a_{i_{1} \ldots i_{m}}$ which has the property

$$
I\left(\ldots, a_{i_{1} \ldots i_{m}}^{\prime}, \ldots\right)=\delta^{p} I\left(\ldots, a_{i_{1} \ldots i_{m}}, \ldots\right)
$$


for some integer $p$, which is called the degree of the invariant $I$. For example, the quadratic form $F=a_{0} x_{1}^{2}+2 a_{1} x_{1} x_{2}+a_{2} x_{2}^{2}$ has an invariant $I=a_{0} a_{2}-a_{1}^{2}$, since one can verify that

$$
I\left(a_{0}^{\prime}, a_{1}^{\prime}, a_{2}^{\prime}\right)=\delta^{2} I\left(a_{0}, a_{1}, a_{2}\right)
$$

A covariant of form $F$ is a polynomial $C$ of the coefficients $a_{i_{1} \ldots i_{m}}$ and variables $x_{1}, \ldots, x_{m}$ which has the property

$$
C\left(\ldots, a_{i_{1} \ldots i_{m}}^{\prime}, \ldots, x_{1}^{\prime}, \ldots, x_{m}^{\prime}\right)=\delta^{p} C\left(\ldots, a_{i_{1} \ldots i_{m}}, \ldots, x_{1}, \ldots, x_{m}\right)
$$

A cubic algebraic curve $f$ is determined by a ternary form of degree three. Its invariants and covariants are clearly studied in [Hilbert, 1993].

Proposition 2 (Hilbert, 1993) The ternary form $f$ corresponding to a planar cubic curve has two basic invariants $S$ and $J$ of degree four and six, respectively, and any other invariant of $f$ is a polynomial of $S$ and $J$. The ternary form $f$ has two covariants of degree three- $f$ and its Hessian $H(f)$, and any other covariant of degree three is a linear combination of $f$ and $H(f)$.

We will not write down explicit formulas for the invariants $S$ and $J$, since they are too long to be fitted in one page. Moreover the formulas are too involved for the computation of $S$ and $J$. Hence we will provide an efficient way to compute $S$ and $J$ for a give cubic curve in the next section.

A ternary form of degree three can be transformed into a normal form by a suitable linear transformation.

Proposition 3 (Walker, 1950) There exists a real linear transformation such that an irreducible real ternary form $f$ of degree three can be transformed into the normal form

$$
f^{\prime}\left(x^{\prime}, y^{\prime}, w^{\prime}\right)=y^{\prime 2} w^{\prime}-x^{\prime}\left(x^{2}+2 a x^{\prime} w^{\prime}+b w^{\prime 2}\right)
$$

where $a$ and $b$ are real constants.

As a consequence of this proposition, an irreducible planar cubic curve can be transformed by a projective transformation into the normal form of (10). This normal form of a cubic curve facilitates the proof of the results in the next section.

\section{Computing Singular Points and Real Inflection Points}

\subsection{Hilbert's solution}

In this section we discuss how to use the basic invariants of a cubic curve $f=0$ to compute the real inflections of $f=0$ by solving only two cubic polynomial equations. Given a planar cubic 
curve $f=0$, consider the family of curves spanned by $f=0$ and $H(f)=0$

$$
g:=\alpha f+6 \beta H(f)=0
$$

Then for any real constants $\alpha$ and $\beta$ that are not both zero, the curve $g=0$ passes through all the intersections between $f=0$ and $H(f)=0$, i.e. the infection points and the singular point (if any) of $f=0$. If we can find $\alpha$ and $\beta$ such that the Hessian curve $H(g)=0$ is the algebraic curve $g=0$ itself, by Proposition $1, g=0$ is reducible to three lines. Thus the problem of finding the singular and inflection points of $f=0$ can be reduced to finding the intersections of three lines with a cubic curve. The existence of such $\alpha$ and $\beta$ is proved by Hilbert.

Proposition 4 (Hilbert, 1993) Let $f=0$ be a planar cubic curve and $H(f)=0$ be its Hessian curve. If $\alpha$ and $\beta$ satisfy

$$
\alpha^{4}-24 S \alpha^{2} \beta^{2}-8 J \alpha \beta^{3}-48 S^{2} \beta^{4}=0
$$

then the curve $g=\alpha f+6 \beta H(f)=0$ degenerates to three lines. Here $S$ and $J$ are the two basic invariants of $f$.

Hilbert's solution provides a complete solution to the problem of finding the inflection points of a cubic curve in the complex projective plane. However, as far as the computation requirement in CAGD is concerned there are still problems that are worthy of further investigation. In the following we first consider how to efficiently obtain equation (12), and hence the basic invariants $S$ and $J$. We then use $S$ and $J$ to classify the singularity type of $f=0$, which determines the number of inflection points of $f=0$. We also investigate how real lines arise as the components of the reducible curve $g=\alpha f+6 \beta H(f)=0$ given by different roots $\alpha: \beta$ of equation (12) and we show that only a special real linear component is needed for computing all the real inflection points of $f=0$.

Since, as mentioned above, the explicit formulas for $S$ and $J$ in terms of the coefficients of $f=0$ are too involved to be used for computing $S$ and $J$, we may get (12) directly as follows. By Proposition 4, there exists a constant $\gamma$ such that

$$
H(\alpha f+6 \beta H(f))=\gamma(\alpha f+6 \beta H(f))
$$

We randomly choose two points $\left(x_{i}, y_{i}, w_{i}\right), i=1,2$ and substitute them into the above equation. Then we can get two equations with $\alpha, \beta, \gamma$ being unknowns. After eliminating $\gamma$, we can arrive at (12). Then the basic invariants $S$ and $J$ can be read off from the coefficients of (12).

For a curve in the normal form (10), the two invariant $S$ and $J$ are simple to compute. 
Theorem 1 The two invariants of a cubic curve in the normal form (10) are given by

$$
S=576\left(4 a^{2}-3 b\right), \quad J=110592 a\left(8 a^{2}-9 b\right)
$$

Proof: For the cubic curve in the normal form

$$
f=y^{2} w-x\left(x^{2}+2 a x w+b w^{2}\right)=0
$$

its Hessian curve is given by

$$
H(f)=8\left(3 x y^{2}+\left(3 b-4 a^{2}\right) x^{2} w+2 a y^{2} w-2 a b x w^{2}-b^{2} w^{3}\right)=0
$$

Setting $(x, y, w)=(1,0,0)$ and $(x, y, w)=(0,0,1)$ on both sides of $(13)$, respectively, we get

$$
\begin{aligned}
\gamma \alpha & =152 \beta\left(20736 b^{2} \beta^{2}+36864 a^{4} \beta^{2}+4 a^{2} \beta^{2}-3 b \beta^{2}-864 a b \alpha \beta-55296 a^{2} b \beta^{2}+768 a^{3} \alpha \beta\right) \\
6 b^{2} \gamma \beta & =-20736 b^{3} \alpha \beta^{2}-1990656 a b^{3} \beta^{3}+1769472 a^{3} b^{2} \beta^{3}+27648 a^{2} b^{2} \alpha \beta^{2}-b^{2} \alpha^{3}
\end{aligned}
$$

Eliminating $\gamma$ from the above equations, we obtain

$$
\alpha^{4}+13824\left(3 b-4 a^{2}\right) \alpha^{2} \beta^{2}+884736 a\left(9 b-8 a^{2}\right) \alpha \beta^{3}-15925248\left(4 a^{2}-3 b\right)^{2} \beta^{4}=0
$$

from which the two invariants $S$ and $J$ can be read off.

\subsection{Singularity detection}

To begin with, we study the real roots of the following equation which is obtained by setting $t=\alpha: \beta$ in (12):

$$
h(t):=t^{4}-24 S t^{2}-8 J t-48 S^{2}=0 .
$$

$h(t)$ is called the characteristic polynomial of the cubic curve $f=0$.

Theorem 2 The distribution of the roots of $h(t)$ is as follows:

1 If $S=J=0$, then $h(t)=0$ has a real root $t=0$ of multiplicity four.

2 If $S=0$ and $J \neq 0$, then $h(t)=0$ has two real roots $t=0$ and $t=2 \sqrt[3]{J}$.

3 If $S \neq 0$ and $J^{2}-64 S^{3}=0$, then $h(t)=0$ has two real roots with opposite signs; one root is $-2 \operatorname{sgn}(J) \sqrt{S}$ of with multiplicity three, and the other root is $6 \operatorname{sgn}(J) \sqrt{S}$ of multiplicity one. 
4 If $S \neq 0$ and $J^{2}-64 S^{3} \neq 0$, then $h(t)=0$ has two simple real roots of opposite signs; furthermore, these two real roots are given by

$$
t_{ \pm}= \pm \sqrt{(12+8 \sqrt{3})|S|}
$$

when $J=0$ or

$$
t_{ \pm}=\operatorname{sgn}(J) \sqrt{2(\gamma+3 S)} \pm \sqrt{2\left(-\gamma+3 S+\frac{|J|}{\sqrt{2(\gamma+3 S)}}\right)}
$$

when $J \neq 0$, where

$$
\gamma=\frac{1}{2} \sqrt[3]{J^{2}-64 S^{3}}-S
$$

Here $t_{+}$is positive and $t_{-}$is negative.

Proof: If $S=0$, then $h(t)=t^{4}-8 J t$ has two real roots $t=0$ and $t=2 \sqrt[3]{J}$. If $S \neq 0$ and $J^{2}-64 S^{3}=0$, let $L=\operatorname{sign}(J) \sqrt{S}$. Then $S=L^{2}$ and $J=8 L^{3}$. It follows that $h(t)=$ $(x-6 L)(x+2 L)^{3}=0$ has two real roots, one with multiplicity one and the other with multiplicity three. In the following we assume $S \neq 0$ and $J^{2}-64 S^{3} \neq 0$.

We construct the Sturm sequence of polynomial $h(t)$ as follows

$$
\begin{aligned}
h_{0} & :=h(t)=t^{4}-24 S t^{2}-8 J t-48 S^{2}, \\
h_{1} & :=h^{\prime}(t) / 4=t^{3}-12 S t-2 J, \\
h_{2} & :=2 S t^{2}+J t+8 S^{2}, \\
h_{3} & :=-\left(J^{2}-64 S^{3}\right) t, \\
h_{4} & :=-S^{2}
\end{aligned}
$$

The sign changes of the Sturm sequence at $t=-\infty, t=0$ and $t=\infty$ are, respectively

$$
\begin{aligned}
& V(-\infty)=V\left(+,-, S, J^{2}-64 S^{3},-\right), \quad V(0)=(-,-J,+,-), \\
& V(+\infty)=V\left(+,+, S,-\left(J^{2}-64 S^{3}\right),-\right)
\end{aligned}
$$

It is easy to verify that $V(-\infty)=3, V(0)=2$ and $V(+\infty)=1$. Hence there exist one root in $(-\infty, 0)$ and one root in $(0, \infty)$. Since $h_{4} \neq 0$, these two roots are simple. Next we derive the expression for the two real roots of $h(t)$.

When $J=0, h(t)=0$ becomes

$$
t^{4}-24 S t^{2}-48 S^{2}=0
$$

Thus the real roots of $h(t)=0$ are

$$
t= \pm \sqrt{12+8 \sqrt{3}|S|}
$$


Now we suppose $J \neq 0$. Introduce a real number $\gamma$ so that the characteristic equation is transformed to

$$
\left(t^{2}+4 \gamma\right)^{2}=8(\gamma+3 S) t^{2}+8 J t+16\left(\gamma^{2}+3 S^{2}\right)
$$

For the righthand side of the above equation to be a perfect square, we need the discriminant to be zero, i.e.

$$
J^{2}-8(\gamma+3 S)\left(\gamma^{2}+3 S^{2}\right)=0
$$

That is

$$
(\gamma+S)^{3}=\left(J^{2}-64 S^{3}\right) / 8
$$

Thus the unique real number $\gamma$ is found to be

$$
\gamma=\frac{1}{2} \sqrt[3]{J^{2}-64 S^{3}}-S
$$

Since $J \neq 0$, it follows from (17) that $\gamma+3 S \neq 0$. So

$$
\left(t^{2}+4 \gamma\right)^{2}=8(\gamma+3 S)\left(t+\frac{J}{2(\gamma+3 S)}\right)^{2}
$$

Taking the square root, we have

$$
t^{2}+4 \gamma= \pm 2 \sqrt{2(\gamma+3 S)}\left(t+\frac{J}{2(\gamma+3 S)}\right)
$$

or

$$
t^{2} \pm 2 \sqrt{2(\gamma+3 S)} t+4 \gamma \pm \frac{2 J}{\sqrt{2(\gamma+3 S)}}=0
$$

The discriminant of this quadratic equation is

$$
\Delta=8\left(-\gamma+3 S \mp \frac{J}{\sqrt{2(\gamma+3 S)}}\right)
$$

Since $h(t)=0$ has only two real roots, we take the $\operatorname{sign} \mp$ in $\Delta$ to be the same as $\operatorname{sgn}(J)$ in order to obtain the two real roots. Thus, by Eqn (18), the real roots of $h(t)=0$ are the roots of

$$
t^{2}-2 \operatorname{sgn}(J) \sqrt{2(\gamma+3 S)} t+4 \gamma-\frac{2|J|}{\sqrt{2(\gamma+3 S)}}=0
$$

with its discriminant

$$
\Delta=8\left(-\gamma+3 S+\frac{|J|}{\sqrt{2(\gamma+3 S)}}\right)>0
$$

It follows that the two real roots of $h(t)=0$ are

$$
t_{+,-}=\operatorname{sgn}(J) \sqrt{2(\gamma+3 S)} \pm \sqrt{2\left(-\gamma+3 S+\frac{|J|}{\sqrt{2(\gamma+3 S)}}\right)}
$$


Note that $t_{+}>0$ and $t_{-}<0$, according to the preceding argument. The theorem is thus proved.

Remark 1: In the above derivation of the expression for the real roots of $h(t)$ in case (4), we use the standard technique in solving a quartic equation in the first step of finding $\gamma$ to make the two sides of (16) perfect squares [Uspensky, 1948]. However, the key to being able to obtain a simple expression for the real roots is that the resolvent cubic equation in $\gamma$ is special enough for us to get a simple expression for $\gamma$, i.e. $\gamma=\frac{1}{2} \sqrt[3]{J^{2}-64 S^{3}}-S$. Note that the expressions for the roots of a general resolvent cubic equation for solving a quartic equation are much more complicated (see again [Uspensky, 1948]).

Remark 2: It will be seen shortly that, in general, only the negative real root of $h(t)=0$ is needed for computing the real inflection points of a cubic curve. The fact that we have obtained an explicit expression for this root in Theorem 4 means that we can compute the real inflection points of a cubic curve without having to solve numerically the quartic equation $h(t)=0$.

Now we consider how the invariants $S$ and $J$ can be used to classify the singularity of a cubic algebraic curve $f=0$. If $S=0$, the Hessian curve $H(f)=0$ consists of three lines, since in this case $t=0$ is a root of $h(t)=0$. The vanishing of $J^{2}-64 S^{3}=0$ signals that $f=0$ is singular. In fact, we have

Theorem 3 An irreducible cubic curve $f=0$ has a singular point if and only if $J^{2}-64 S^{3}=0$. If $f=0$ is singular, its singular point is always real and the singular point is a crunode, a cusp, or an acnode if and only if $J>0, J=0$, or $J<0$, respectively.

Proof: By Proposition 3, any cubic curve can be transformed into the normal form (10) by a suitable real projective transformation, and the type of its singular points and the number of its inflection points are not changed by the transformation. Accordingly, the two invariants $S$ and $J$ are transformed to $S^{\prime}=\delta^{4} S$ and $J^{\prime}=\delta^{6} J$, and hence $\left(J^{\prime}\right)^{2}-64\left(S^{\prime}\right)^{3}=\delta^{12}\left(J^{2}-64 S^{3}\right)$, where $\delta \neq 0$ is the determinant of the transformation matrix. Thus we just need to prove the statement for a cubic curve in the normal form (10). Note that here we just make use of the existence of the normal form of a cubic curve but do not need to actually compute it.

The canonical curve (10) has a singular point if and only if $b=0$ or $a^{2}-b=0$, and the singular point is real $((0,0,1)$ or $(-a, 0,1))$. Thus the singular point of a real singular cubic curve is real.

On the other hand, by Theorem 1, we have

$$
J^{2}-64 S^{3}=\left(110592 a\left(8 a^{2}-9 b\right)\right)^{2}-64\left(576\left(4 a^{2}-3 b\right)\right)^{3}=-2^{24} 3^{9} b^{2}\left(a^{2}-b\right)
$$

Thus the canonical curve (10) has a singular point if and only if $J^{2}-64 S^{3}=0$. 
Now assume $J^{2}-64 S^{3}=0$, that is, $b=0$ or $a^{2}-b=0$. Obviously, $S=J=0$ if and only if $a=b=0$, and if and only if the singular point is a cusp. Next we assume $b=0$ but $a \neq 0$, so $S=2304 a^{2}$ and $J=884736 a^{3}$. In this case, the canonical curve (10) becomes $y^{2}=x^{2}(x+2 a)$. It has a crunode if and only if $a>0$, and if and only if $J>0$. It has an acnode if and only if $a<0$, and if and only if $J<0$. For the case $a^{2}-b=0$ but $a \neq 0$, one can show similarly that the same results hold.

Theorem 4 If an irreducible cubic curve $f=0$ is singular, then $H(f)=0$ is also singular; furthermore, in this case the singular point of $f=0$ is a singular point of $H(f)=0$.

Proof: Again we just need to prove that the statement holds for an irreducible cubic curve $f=0$ in the normal form (10). It can be computed that the two invariants of the Hessian curve $H(f)=0$ are given by

$S^{\prime}=2^{18}\left(576 a^{6}-1296 a^{4} b+729 b^{3}\right), \quad J^{\prime}=2^{30} 3^{3} a\left(512 a^{8}-1728 a^{6} b+3888 a^{4} b^{2}-4860 a^{2} b^{3}+2187 b^{4}\right)$

Thus

$$
J^{\prime 2}-64 S^{\prime 3}=2^{60} 3^{12}\left(a^{2}-b\right) b^{2}\left(4 a^{2}-3 b\right)^{6}=2^{36} 3^{3}\left(4 a^{2}-3 b\right)^{6}\left(J^{2}-64 S^{3}\right)
$$

Since $f=0$ is singular, by Theorem $3, J^{2}-64 S^{3}=0$. It follows from (19) that $J^{\prime 2}-64 S^{\prime 3}=0$. Then, by Theorem $3, H(f)=0$ is singular.

For the cubic curve $f=0$ in the normal form

$$
f=y^{2} w-x\left(x^{2}+2 a x w+b w^{2}\right)=0
$$

its Hessian curve is

$$
H(f)=8\left(3 x y^{2}+\left(3 b-4 a^{2}\right) x^{2} w+2 a y^{2} w-2 a b x w^{2}-b^{2} w^{3}\right)=0
$$

If $f=0$ is singular, then by Theorem 1 and Theorem 3 , either $b=0$ or $a^{2}-b=0$, with the singular point of $f=0$ at $(0,0,1)$ or $(-a, 0,1)$, respectively. It is then straightforward to verify that $(0,0,1)$ or $(-a, 0,1)$ is also a singular point of $H(f)=0$ when $b=0$ or $a^{2}-b=0$, respectively.

\subsection{Computing a real line that contains all real inflection points}

From now on we set $\beta=1$ in a root $t=\alpha: \beta$ of Eqn (15). For each solution $t=\alpha$ of equation (15), the corresponding curve $g=\alpha f+6 H(f)=0$ can be factored into three lines. We need to know how many of the three lines are real. 
Theorem 5 Let $\alpha$ be a real root of $h(t)=0$. Then the curve $g=\alpha f+6 H(f)=0$ consists of three distinct real lines if and only if $\alpha>0$ or $\alpha=0$ and $J<0$, and contains one real line if and only if $\alpha<0$ or $\alpha=0$ and $J>0$. In particular, when $S=J=0, g=H(f)$ consists of three real lines, and one of them is a double line.

Proof: We assume again that the cubic curve $f=0$ under consideration takes the normal since any real irreducible cubic curve can be transformed to the normal form by a real projective transformation, which does not change the number of real components of the curve $g=0$ that is derived from $f=0$; furthermore, a double line is transformed to a double line. On the other hand, the characteristic polynomial $h(t)$ is transformed to

$$
h^{\prime}(t)=t^{4}-24 S \delta^{4} t^{2}-8 J \delta^{6} t-48 S^{2} \delta^{8}=0
$$

where $\delta$ is the determinant of the transformation matrix. Thus the roots of $h^{\prime}(t)=0$ differ from the roots of $h(t)$ only by a positive factor $\delta^{2}$.

For $f=0$ in the normal form (10), direct computation yields

$$
\begin{aligned}
g & =\alpha f+6 H(f) \\
& =-\alpha x^{3}+\left(144 b-2 \alpha a-192 b^{2}\right) x^{2} w+144 x y^{2}+(-96 a v-\alpha b) x w^{2}+(\alpha+96 a) y^{2} w-48 b^{2} w^{3}
\end{aligned}
$$

When $\alpha \neq 0$, the curve $g=0$ intersects the line at infinity $w=0$ in three distinct points $(0,1,0)$, $(\sqrt{\alpha} x, 12,0)$ and $(-\sqrt{\alpha} x, 12,0)$. If $\alpha>0$, the three intersection points are real, so $g=0$ consists of three distinct real lines; if $\alpha<0$, two of the intersection points are complex conjugate, so $g=0$ contains only one real line.

When $\alpha=0$ is a root of $h(t)=0, S$ must be zero, so $b=4 a^{2} / 3$ and $J=-442368 a^{3}$. Then the curve

$$
g=(3 x+2 a w)\left(9 y^{2}-8 a^{3} w^{2}\right)=0
$$

degenerates to three real lines if and only if $a>0$, and if and only if $J<0$. Similarly, $g=0$ contains one real line if and only if $J>0$.

When $S=J=0, \alpha=0$ is the only root of $h(t)=0$, and $g=6 H(f)=24 x y^{2}$. Thus $g=0$ consists of three real lines, one of which is a double line.

Theorem 6 The number of inflection points of an irreducible cubic curve $f=0$ is as follows:

1 If $f=0$ has a cusp, then $f=0$ has one real inflection point;

2 If $f=0$ has a crunode, then $f=0$ has one real inflection point and a pair of complex conjugate inflection points; 
3 If $f=0$ has an acnode, then $f=0$ has three real inflection points;

4 If $f=0$ is nonsingular, then $f=0$ has three real inflection points and three pairs of complex conjugate inflection points.

Proof: If $f=0$ has a cusp, by Theorem $3, S=J=0$. Hence $f=y^{2} w-x^{3}$ and $H(f)=24 x y^{2}$. The curves $f=0$ and $H(f)=0$ have two distinct intersection points: the singular point $(0,0,1)$ of $f=0$ of multiplicity 8 and the inflection point $(0,1,0)$ of $f=0$.

When $J^{2}-64 S^{3}=0$ but $S \neq 0, f=0$ has a crunode or an acnode. In this case, $b\left(a^{2}-b\right)=0$, so $b=0$ or $a^{2}-b=0$. We consider only the case $b=0$ since the argument is similar when $a^{2}-b=0$. Then $(0,0,1)$ is the singular point of $f=y^{2}-x^{2}(x+2 a w)=0$. The two real roots of $h(t)=0$ are $\alpha_{1}=-96 a$ and $\alpha_{2}=288 a$, which yield

$$
g_{1}=\alpha_{1} f+6 H(f)=48 x\left(2 a x^{2}+3 y^{2}\right), \quad g_{2}=\alpha_{2} f+6 H(f)=-48(3 x+8 a w)\left(-y^{2}+2 a x^{2}\right)
$$

It is easy to see that $g_{1}=0$ and $g_{2}=0$ intersect in four points $(-8 a / 3, \pm 8 / 9 \sqrt{-6 a} a, 1),(0,0,1)$, and $(0,1,0)$. The point $(0,0,1)$ is the singular point of $f=0$ whose multiplicity is 6 . The other three points are the inflection points. If $a>0$ (i.e. $J>0$, or $f=0$ has a crunode), one inflection point is real and the other two are complex conjugate; if $a<0$ (i.e. $J<0$, or $f=0$ has an acnode), the three inflection points are real.

When $J^{2}-64 S^{3} \neq 0, f=0$ is nonsingular. We consider two cases: $S=0$ and $S \neq 0$. If $S=0$, then $f=y^{2}-x\left(x^{2}+2 a x+4 / 3 a^{2}\right)$ and $H(f)=8 / 9\left(9 y^{2}-8 a^{3} w^{2}\right)(3 x+2 a w)$. The two roots of $h(t)=0$ are $\alpha_{1}=0$ and $\alpha_{2}=-96 \sqrt[3]{4}$. Thus

$$
\begin{aligned}
g_{1} & =6 H(f)=16 / 3\left(9 y^{2}-8 a^{3} w^{2}\right)(3 x+2 a w) \\
g_{2} & =\alpha_{2} f+6 H(f) \\
& =4 / 9 c(3 x+2(1-c) a w)\left(72 a x^{2}+48(2+c) a^{2} x w+8(c+2)^{2} a^{3} w^{2}+27 c^{2} y^{2}\right)
\end{aligned}
$$

where $c=\sqrt[3]{4}$. It can be directly computed that the nine intersection points of $g_{1}=0$ and $g_{2}=0$, i.e. the inflection points of $f=0$, are $(0,1,0),(-2 / 3 a, \pm 2 / 9 a \sqrt{-6 a}, 1),(2 / 3(c-$ 1) $a, \pm 2 / 3 a \sqrt{2 a}, 1)$ and $((-(2+c) a \pm c \sqrt{-3 a}) / 3, \pm 2 / 3 a \sqrt{2 a}, 1)$. If either $a>0$ or $a<0$, there are three real points and three pairs of complex conjugate points among the nine intersection points.

If $S \neq 0$, by Theorem $2, h(t)=0$ has two real roots and a pair of conjugate roots $\lambda \pm \mu$ $(\mu \neq 0)$, where $\lambda$ is real and $\mu \neq 0$ is a pure imaginary number. The intersection of the two complex curves $g_{ \pm}=(\lambda \pm \mu) f+6 H(f)$ also gives the nine inflection points of $f=0$. Let

$$
g_{+}=l_{1} l_{2} l_{3}, \quad g_{-}=\bar{l}_{1} \bar{l}_{2} \bar{l}_{3}
$$


where $l_{i}, i=1,2,3$ are linear functions. Each line $l_{i}$ is not a real line, for otherwise $g_{+}$and $g_{-}$ would have a common linear component, contradicting that $f=0$ is irreducible (ref. Proposition 1). Each line $l_{i}$ and its conjugate $\bar{l}_{i}$ intersect at a real point. We now claim that $l_{i}$ and $\bar{l}_{j}(i \neq j)$ intersect at an imaginary point. If, on the contrary, the intersection of $l_{i}$ and $\bar{l}_{j}$ is a real point $P_{0}$, then $\bar{l}_{i}$ and $l_{j}$ also intersect at $P_{0}$. Then $P_{0}$ is a singular point of $g_{+}$since two of its components $l_{i}$ and $l_{j}$ pass through $P_{0}$. Similarly, $P_{0}$ is also a singular point of $g_{-}$. Thus $P_{0}$ is a singular point of the curve $f=\left(g_{+}-g_{1}\right) /(2 \mu)=0$. But this contradicts that $f=0$ is nonsingular. Hence, when $S \neq 0, f=0$ has three real inflection points which are the intersections of $l_{i}$ and $\bar{l}_{i}, i=1,2,3$, and three pairs of complex conjugate inflection points which are the remaining intersections between $f=0$ and $H(f)=0$.

Remark: The results of Theorem 6 regarding the number of inflection points for different types of singular planar cubic curves is well known in algebraic geometry (see, e.g. [Gibson, 1998]). But we give a new proof which relates the different cases to the two invaiants $S$ and $J$, thus providing a basis for our algorithm to be described below.

Before giving the algorithm, we need the following two corollaries for preparation.

Corollary 7: Given an irreducible planar cubic curve $f=0$, there exists a unique real root $\alpha$ of $h(t)=0$ such that $g=\alpha f+H(f)=0$ has exactly one simple real linear component. Specifically, if $S=J=0$, i.e. $f=0$ has a cusp, the only root $\alpha=0$ of $h(t)=0$ gives $g:=H(f)=0$ which has a simple real linear component and a real double linear component; if $S \neq 0$ or $J \neq 0$, there exists a root $\alpha$ of $h(t)=0$ such that $g=\alpha f+H(f)=0$ has one simple real linear component and a pair of complex conjugate components.

Proof: The corollary follows from Theorem 2 and Theorem 5 by the following choices of the root $\alpha$ in different cases: When $S=J=0$ (case (1) of Theorem 2), choose $\alpha=0$. When $S=0$ and $J>0$ in case (2) of Theorem 2, choose $\alpha=0$. In cases (3) and (4) or when $S=0$ and $J<0$ in case (2) of Theorem 2, choose $\alpha$ to be the only negative root of $h(t)=0$ as given in Theorem 2 .

Let $l$ denote the unique real simple linear component of the curve $g=\alpha f+H(f)=0$ as prescribed in Corollary 7.

Corollary 8: If $f=0$ has three real inflection points, i.e. $f=0$ is nonsingular or has an acnode, then the real line $l$ contains all the three real inflection points. When $f=0$ has a crunode or a cusp, $l$ contains the only real inflection of $f=0$ and the crunode or cusp.

Proof: First suppose that $f=0$ has three real inflection points. Then, by Theorem $6, f=0$ does not have a cusp; hence, by Theorem $3, S \neq 0$ or $J \neq 0$. Then, by Corollary 7 , there exists a unique real root $\alpha$ of $h(t)=0$ such that $g=\alpha f+H(f)=l l_{1} \bar{l}_{1}$, where $l$ is a simple real linear factor and $l_{1}, \bar{l}_{1}$ are a pair of complex conjugate lines. Note that, as an imaginary line, 
$l_{1}$ or $\bar{l}_{1}$ cannot contain two distinct real points. Clearly, $l$ contains at least one real inflection point $r_{1}$ of $f=0$. Suppose the other two real inflection points $r_{2}$ and $r_{3}$ are not on $l$, but on $l_{1}$ and $\bar{l}_{1}$, respectively. Let $q$ denote the real intersection point of $l_{1}$ and $\bar{l}_{1}$. Then either $q \neq r_{2}$ or $q \neq r_{3}$. But this contradicts that both $l_{1}$ and $\bar{l}_{1}$ are imaginary lines. Hence, all the three inflection points of $f=0$ are on the real line $l$.

When $f=0$ has a crunode or a cusp, the proof is given by the proof for cases (1) and (2) in Theorem 6 .

\subsection{Algorithm for computing real inflection points}

Based on the preceding analysis, we present the algorithm for computing the real inflection points and the singular point (if any) of an irreducible cubic algebraic curve $f=0$.

Algorithm: Real-Inflections

Input: An irreducible real cubic algebraic curve $f=0$.

Output: The real inflection points and singular point (if any) of $f=0$.

BEGIN

Step 1 Compute the Hessain curve $H(f)=0$ of the curve $f=0$.

Step 2 Obtain the characteristic polynomial $h(t)$ as defined in (15), using the procedure described after Proposition 4 in Section 2. Extract the invariants $S$ and $J$ from the coefficients of Eqn. (15).

Step 3 Compute the real roots of $h(t)=0$. If $h(t)=0$ has only one root $\alpha_{1}=0$, then find the simple real linear component $l$ of $H(f)=0$. Otherwise, let $\alpha_{1}<\alpha_{2}$ be the two real roots of $h(t)=0$. Then, by Corollary $7, g_{1}=\alpha_{1} f+6 H(f)=0$ contains only one simple real line.

Factor out this line $l$ as follows. Choose two $x$ values $x_{1}$ and $x_{2}$, and substitute them into $g_{1}=0$ to solve for the simple real values $y_{1}, w_{1}$ and $y_{2}, w_{2}$ such that $g_{1}\left(x_{i}, y_{i}, w_{i}\right)=0$, $i=1,2$. This requires to find the real roots of two cubic equations which are known to contain only one simple real root. Then the line $l=0$ passing through $\left(x_{1}, y_{1}, w_{1}\right)$ and $\left(x_{2}, y_{2}, w_{2}\right)$ is the simple real line of $g_{1}=0$.

Step 4 Parameterize line $l: l=l(u)$. Substitute $l(u)$ into $f=0$ (or equivalently, $g_{2}=0$ given by the other real root of $h(t)=0$ if $h(t)$ has two real roots) to get a cubic polynomial in $u$. By Corollary $8, g_{2}(l(u))=0$ has three real roots; a simple root corresponds to a real inflection point, and a double root (if any) corresponds to a singular point of $f=0$. 
The case of $J^{2}-64 S^{3}=0$ and $J<0$, i.e. $f=0$ has an acnode, should be treated separately if one also wants to find the acnode. In this case, the above intersection of $l=0$ with $f=0$ yields the three real inflection points of $f=0$. To find the acnode, we first obtain the quadratic factor $p=g_{1} / l$, which consists of a pair of conjugate lines, and the intersection of these two lines gives the acnode of $f=0$. This intersection point can be computed by solving the linear system of equations: $p_{x}^{\prime}=p_{y}^{\prime}=p_{w}^{\prime}=0$.

\section{END}

The above algorithm can also be adapted to detect if there exist a singular point and/or inflection points in a region $R$ in the plane. Suppose first that the region $R$ is convex for the sake of simplicity in discussion. In Step 4, instead of solving the equation $g_{2}(l(u))=0$, we can check if the cubic polynomial $g_{2}(l(u))=0$ has any real roots in some interval $u \in[a, b]$ determined by the part of the line $l$ intercepted within the region $R$. If $g_{2}(l(u))=0$ has a simple root in $u \in[a, b]$, then $f=0$ has an inflection point in $R$, and if $g_{2}(l(u))=0$ has a double root in $u \in[a, b]$, then $f=0$ has a singular point, i.e. a crunode or a cusp, in $R$; a separate checking is needed for an acnode. When the region $R$ is nonconvex, one may need to check against several intervals of $u$ since more than one segment on the line $l(u)$ may fall inside $R$.

\subsection{Examples}

We use three examples to explain how the algorithm works.

Example 1 Given a cubic algebraic curve

$$
f:=-8 x^{3}-12 x^{2} y-6 x y^{2}-y^{3}-7 x^{2} w-2 x y w+7 y^{2} w-2 x w^{2}-y w^{2}
$$

We are going to find the singular point and real inflection points of $f=0$. It is easy to compute that $S=360000, J=-1728000000$ and $J^{2}-64 S^{3}=0$. Thus $f=0$ has an acnode and three real inflection points. The two real roots of the characteristic polynomial

$$
h(t)=t^{4}-8640000 t^{2}+13824000000 t-6220800000000
$$

are $\alpha_{1}=-3600$ and $\alpha_{2}=1200$, and the corresponding curves are

$$
\begin{aligned}
& g_{1}=-1200\left(-30 x^{3}-75 x^{2} y-90 x y^{2}-30 y^{3}-19 x^{2} w-14 x y w+4 y^{2} w-2 x w^{2}-y w^{2}+w^{3}\right) \\
& g_{2}=-1200\left(+2 x^{3}-27 x^{2} y-66 x y^{2}-26 y^{3}+9 x^{2} w-6 x y w-24 y^{2} w+6 x w^{2}+3 y w^{2}+w^{3}\right)
\end{aligned}
$$

By substituting $x=0$ and $w=1$ into $g_{1}=0$, we get two points on the curve $g_{1}=0$ : $P_{1}=$ $(0,1 / 3,1)$ and $P_{2}=(1,-5 / 3,1)$. The line $l=6 x+3 y-w=0$ passing through $P_{1}$ and $P_{2}$ is the 
only real linear component of $g_{1}=0$. To find the inflection points, we intersect $l$ with $g_{2}=0$, and find three real inflection points: $(1,-2,0),(9+4 \sqrt{3}, 3-8 \sqrt{3}, 45)$ and $(9-4 \sqrt{3}, 3+8 \sqrt{3}, 45)$.

To further find the acnode of $f=0$, let $p=g_{1} / l=-5 x^{2}-10 x y-10 y^{2}-4 x w-2 y w-w^{2}$. The singular point is the solution of the linear system of equations

$$
p_{x}^{\prime}:=-10 x-10 y-4 w=p_{y}^{\prime}:=-10 x-20 y-2 w=p_{w}^{\prime}:=-4 x-2 y-2 w=0
$$

which is $(-3,1,5)$.

Example 2 Let the cubic algebraic curve be

$$
f:=-x^{3}+6 x^{2} y-12 x y^{2}+8 y^{3}-8 x^{2} w+38 x y w-35 y^{2} w-25 x w^{2}+56 y w^{2}-26 w^{3}
$$

One can compute that $S=J=0$, thus $f=0$ has a cusp and one real inflection point. In this case $h(t)=0$ has only one root $\alpha=0$, leading to $g=\alpha f+H(f)=H(f)$, which is

$$
H(f)=216\left(x^{3}-3 x y^{2}-2 y^{3}+5 x^{2} w+4 x y w-y^{2} w+7 x w^{2}+4 y w^{2}+3 w^{3}\right)
$$

We now choose $x=0, w=1$ and substitute them into $H(f)=0$ to get two simple real points on $H(f): P_{1}=(0,3,2)$ and $P_{2}=(1,2,1)$. The line $l=x-2 y+3 w=0$ passing through $P_{1}$ and $P_{2}$ is the simple real line of $H(f)$. We substitute $x=2 y-3 w$ into $f=0$ and find a simple root $y: w=1: 0$ and a double root $y: w=2: 3$. Thus we get the real inflection point $(2,1,0)$ and the cusp $(-5,2,3)$.

Example 3 The algebraic cubic curve is given by

$$
f:=x y^{2}-(x-w)(2 x-w) w
$$

We are going to detect if there exist any inflection points of $f=0$ in the rectangular region $R=[0,1] \times[0,1]$.

For the given curve $f=0, J^{2}-64 S^{3}=-330225942528<0$, thus $f=0$ is nonsingular and thus has three real inflection points. The two real roots of the characteristic polynomial $h(t)$ are $\alpha_{1}=-48 \sqrt{9+6 \sqrt{3}}$ and $\alpha_{2}=48 \sqrt{9+6 \sqrt{3}}$, and the corresponding two curves are

$$
\begin{aligned}
& g_{1}=-48\left(4 x^{3}+(p+3) x y^{2}-2(p+3) x^{2} w-3 y^{2} w+3(p+1) x w^{2}-p w^{3}\right) \\
& g_{2}=-48\left(4 x^{3}-(p-3) x y^{2}+2(p-3) x^{2} w-3 y^{2} w-3(p-1) x w^{2}-p w^{3}\right)
\end{aligned}
$$

where $p=\sqrt{9+6 \sqrt{3}}$.

The only real line component of $g_{1}$ is $l=6 x-(p-3) \sqrt{3} w . l=0$ intersects the rectangular region $R$ at two points $P_{1}=((p-3) \sqrt{3} / 6,0,1)$ and $P_{2}=((p-3) \sqrt{3} / 6,1,1)$. We parameterize $l$ by $l=(1-u) P_{1}+u P_{2}=0$, and substitute it into $g_{2}=0$ :

$$
g_{2}(l(u))=1 / 3((p-3) \sqrt{3}-6)\left(3 u^{2}-(p-3) \sqrt{3}-p+6\right)
$$


One can check $g_{2}(l(u))=0$ has one simple root in $[0,1]$. Thus $f=0$ has one real inflection point in the region $R$.

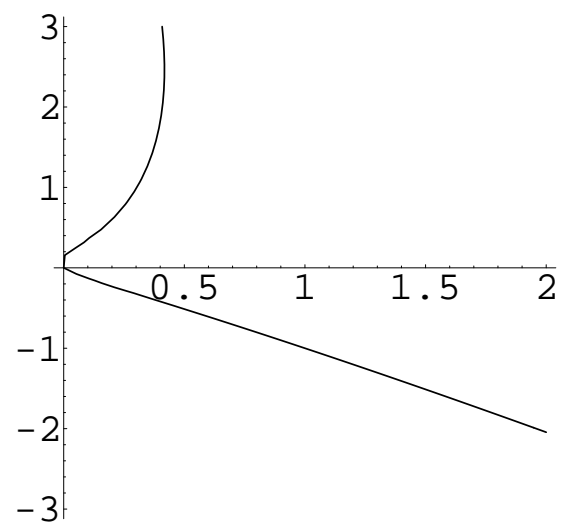

Figure 1: The cubic curve in Example 1.

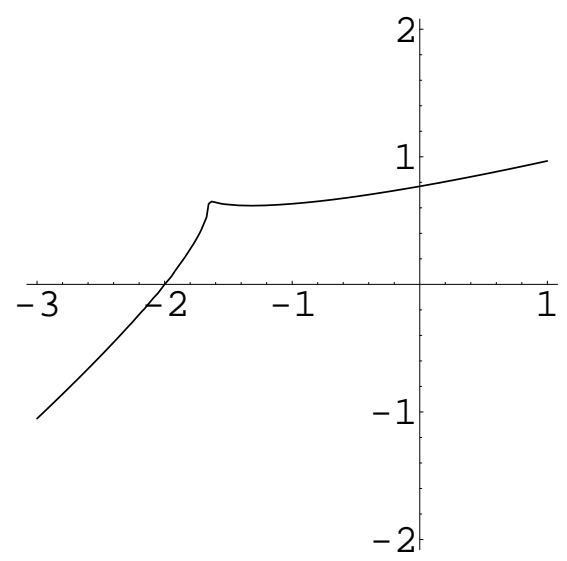

Figure 2: The cubic curve in Example 2.

\section{Acknowledgment}

The authors would like to thank Richard Patterson, Barry Joe, and Ron Goldman for helpful discussions.

Falai Chen is supported by the National Natural Science Foundation of China (19971087), NKBRSF on Mathematical Mechanics (grant G1998030600) and a grant for Distinguished Young Teachers from Educational Ministry of China. Wenping Wang is supported partially by a grant (HKU 7032/00E) from Research Grant Council of Hong Kong. 


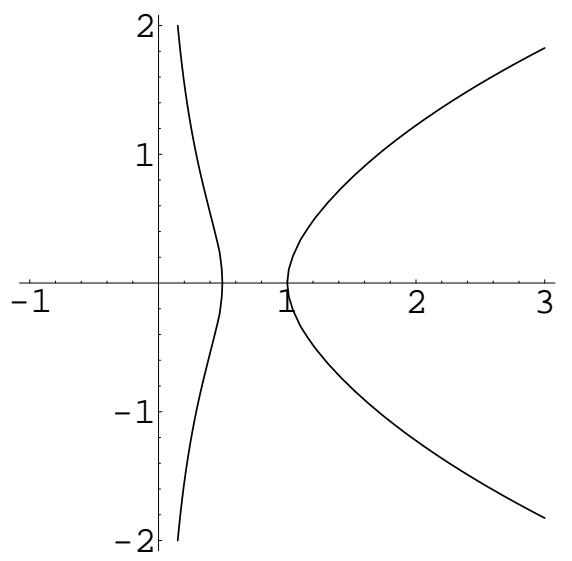

Figure 3: The cubic curve in Example 3.

\section{References}

[Abhyankar and Bajaj, 1987] S.S. Abhyankar and C. Bajaj (1987), Automatic parameterization of rational curves and surfaces II: cubics and cubicoids, Computer-Aided Design, vol. 19, no. 9 , pp. 499-502.

[Gibson, 1998] C.G. Gibson, Elementary Geometry of Algebraic Curves, Cambridge University Press, 1998.

[Hilbert, 1993] D. Hilbert, Theory of Algebraic Invariants, Translated by R. C. Laubenbacher. Cambridge University Press, 1993.

[Li and Cripps, 1997] Y.M. Li and R.J. Cripps (1997), Identification of inflection points and cusps on rational curves, Computer Aided Geometric Design, vol. 14, pp. 491-497.

[Manocha and Canny, 1992] D. Manocha and J. Canny (1992), Detecting cusps and inflections in curves, Computer Aided Geometric Design, vol. 1, pp. 1-24.

[Olver, 1999] Olver, P.J. Classical Invariant Theory, Cambridge University Press, 1999.

[Paluszny and Patterson, 1998] M. Paluszny and R. Patterson (1998), Geometric control of $G^{2}$ cubic A-splines, Computer Aided Geometric Design, vol. 15, pp. 261-287.

[Patterson, 1988a] R.R. Patterson (1988), Parameterizing and graphing non-singular cubic curves, Computer-Aided Design, vol. 20, no. 10, pp. 615-623.

[Patterson, 1988b] R.R. Patterson (1988), Parametric cubics as algebraic curves, Computer Aided Geometric Design, vol. 5, pp. 139-159. 
[Stone and DeRose, 1989] M.C. Stone and T.D. DeRose (1989), A geometric characterization of parametric cubic curves, ACM Transactions on Graphics, vol. 8, pp. 147-163.

[Uspensky, 1948] J.V. Uspensky, Theory of Equations, McGraw-Hill Book Company, Inc., New York, 1948.

[Wang, 1981] J.Y. Wang (1981), Shape classification of the parametric cubic curve and parametric B-spline cubic curve. Computer-Aided Design, vol. 13, pp. 199-206.

[Wang et al, 2002] W. Wang, B. Joe, and R.N. Goldman (2002), Computing quadric surface intersection based on an analysis of planar cubic curves, 2002, submitted.

[Walker, 1950] R.J. Walker, Algebraic Curves, Princeton University Press, 1950. 